\title{
Le problème de Cauchy pour une équation linéaire à coefficients polynomiaux
}

Sommario delle conferenze tenute nei giorni 22-23-24 maggio 1956

On se donne une équation aux dérivées partielles linéaire, à coefficients polynomiaux, de type quelconque. Le problème de Cauchy, à données nulles, est résolu par une transformation fonctionnelle, définie par une quadrature et opérant sur la solutions d'un problème de Cauchy particulier: la transformation fonctionnelle ne dépend que de la surface portant les donnees de Cauchy du premier problème; le second problème de Cauchy ne dépend que de l'équation donée; l'ordre de l'une des équations est le degré des coefficients de l'autre: si l'equation donnee est à coefficients linéaire sa résolution est donc réduite à des quadratures et à l'intégration d'un système différentiel ordinaire. On peut faire en sorte que la seconde équation suit la transformée formelle de Laplace de la première; les caractéristiques de l'une sont transformées de Legendre de celles de l'antre; elles ont mêmes bicaractéristiques.

La transformation fonctionnelle útilisée peut être definie à l'aide de la trace fonctionnelle da L. Fantappiè, à condition d'en élargir un peu la définition, et d'une integrale triple.

Poúr plus de détails, voir: Comptes Rendus, Paris, 242, 1956, p. 953- 959. 